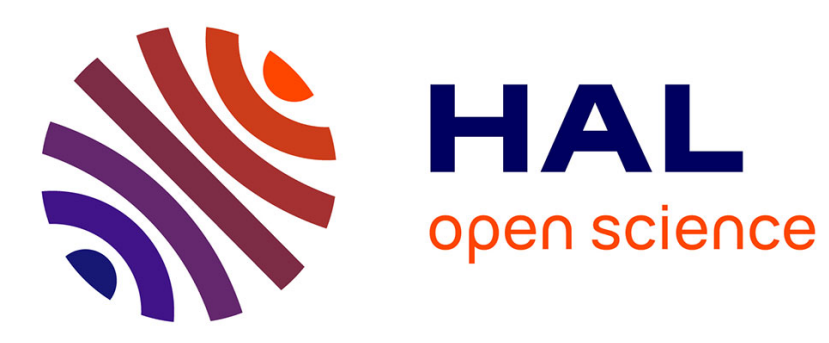

\title{
Nanostructures Defined by The Local Oxidation of Ferromagnetic GaMnAs Layer
}

Voves J. Voves J., Zbynek Soban, Michal Jelinek, Volodimir Komarnickij, Miroslav Cukr, Vit Novak

\section{- To cite this version:}

Voves J. Voves J., Zbynek Soban, Michal Jelinek, Volodimir Komarnickij, Miroslav Cukr, et al.. Nanostructures Defined by The Local Oxidation of Ferromagnetic GaMnAs Layer. ENS 2007, Dec 2007, Paris, France. pp.85-90. hal-00202518

\section{HAL Id: hal-00202518 \\ https://hal.science/hal-00202518}

Submitted on 7 Jan 2008

HAL is a multi-disciplinary open access archive for the deposit and dissemination of scientific research documents, whether they are published or not. The documents may come from teaching and research institutions in France or abroad, or from public or private research centers.
L'archive ouverte pluridisciplinaire HAL, est destinée au dépôt et à la diffusion de documents scientifiques de niveau recherche, publiés ou non, émanant des établissements d'enseignement et de recherche français ou étrangers, des laboratoires publics ou privés. 


\title{
NANOSTRUCTURES DEFINED BY THE LOCAL OXIDATION
}

OF FERROMAGNETIC GaMnAs LAYER

\author{
J. Voves, Z. Šobáň, M. Jelínek, V. Komarnickij \\ Department of Microelectronics, The Czech Technical University FEE Prague, \\ Technická 2, CZ-166 27 Praha 6, Czech Republic
}

M. Cukr, V. Novák

Department of Surfaces and Interfaces, The Institute of Physics of the ASCR v.v.i., Cukrovarnická 10, CZ-162 53 Praha 6, Czech Republic

\begin{abstract}
The results of Local Anodic Oxidation (LAO) on the thin GaMnAs layers are reported. The ferromagnetic GaMnAs layers were prepared by low temperature MBE growth in a Veeco Mod Gen II machine. The LAO process was performed with the AFM microscope Smena NT-MDT placed in the sealed box with the controlled humidity in the range $45-80 \%$. The oxide was grown in the semicontact mode of the AFM. Sample was positively biased with respect to the AFM tip with the bias from 6 to $24 \mathrm{~V}$. The conductive diamond coated AFM tips with the radius $30 \mathrm{~nm}$ were utilized for the oxidation. The tip speed during the oxidation was changed from $400 \mathrm{~nm} / \mathrm{s}$ to $1.5 \mu \mathrm{m} / \mathrm{s}$. The tip force was also changed during the oxidation. The height of oxide nanolines increases with applied voltage from 3 to $18 \mathrm{~nm}$. The width of these lines was approximately $100 \mathrm{~nm}$ at half of the maximum.
\end{abstract}

\section{INTRODUCTION}

The fabrication and study of nanoelectronic devices demands the modification of metals and semiconductors on the nanometer length scale. Key elements for more complex structures such as transistors are narrow conducting wires and tunneling barriers. On the nanometer scale proximal probe based instruments, in particular the scanning tunneling microscope (STM) and atomic force microscope (AFM), have proven successful not only to image surfaces but also to modify them. AFM is well-known instrument used for surface topography measurements. Principle of the method lies in monitoring

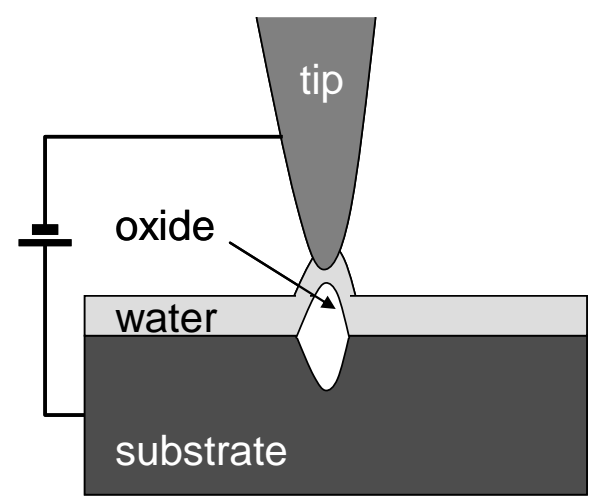

Fig. 1 Local anodic oxidation by the AFM tip

of the tip motion above the inspected surface. The tip is fixed on the end of elastic cantilever, which is deflected by attractive and repulsive forces between the surface and the tip. The deflection of the cantilever is optically scanned. Various approaches have been used to generate patterns by means of the AFM useful for device fabrication: mechanically modifying a resist layer that then acts as an etch mask for pattern transfer, local anodic oxidation and subsequent use of the oxide as the etch mask, and maskless techniques, i.e., those directly affecting the material's electron system by the proximal probe itself. This can be done again mechanically or by using the probe for locally inducing chemical reactions. Early work on STM and contact AFM induced oxidation of Si was done by Dagata [1]. If the substrate surface and the tip are electrically conductive and the tip is biased negatively to the surface, local oxidation proceeds under the tip (see Fig.1). With this method it's possible to fabricate oxide lines of any shape. Height of these 


\section{J. Voves, Z. Šobán̆, M. Jelínek, V. Komarnicki, M. Cukr, V. Novák \\ Nanostructures defined by the local oxidation of ferromagnetic GaMnAs layer}

formations reaches several nanometers and their width at half of the maximum is about $100 \mathrm{~nm}$. Oxide lines can be written to form desired patterns on the surface by translating the tip in a controlled fashion. Several research groups have demonstrated the viability of this technique in both contact and tapping AFM modes. However, when LAO is performed in contact mode, the tip apex tends to degrade readily since the tip stays in physical contact with the surface. This subsequently shortens the life-time of the tip and sacrifices the resolution of oxide patterns. For these reasons, the noncontact/tapping AFM mode is the most preferred to perform LAO. The AFM tip oxidizes the surface and forms an energy barrier for electrons in the metal or in the heterostructure. The LAO patterning is broadly reported on the metal (Ti) [2], Si [3] and GaAs layers [4,5]. Unsuccessful attempt has been realized with LAO on the GaN layer [6].

This method could be advantageous especially for the laboratory experiments. The sample surface can be examined before the oxidation. Consequently it's possible to create oxide lines on the selected location. Oxidation results can be immediately investigated. In this work, a conducting AFM tip is used in tapping mode to oxidize GaMnAs surface producing nanometric constrictions in the thin ferromagnetic layer.

Several spin-valve and spin-filtering effects have been reported in the ferromagnetic nanostructures with the domain-wall pinning on the nanoconstrictions. The giant magnetoresistance (GMR) up to several hundred \% is produced by the opposite magnetisation of adjacent domain walls, the tunnel magnetoresistance (TMR) appears, when the constrictions are very narrow (sub 10 $\mathrm{nm}$ ), producing up to several thousand \% [7]. Tunneling anisotropic magnetoresistance (TAMR) is present in the nanoconstricted structures with the strong spin-orbit coupling [8]. Application of the external magnetic field in the different directions produces both positive and negative MR. Most of nanoconstricted ferromagnetic structures are based on the GaMnAs layers patterned by the electron beam lithography. The LAO by AFM is attracting attention because of its relatively low cost and high resolution. Very few reports are presented about the $\mathrm{LAO}$ on the ferromagnetic GaMnAs layers.

\section{EXPERIMENTAL}

We present some results of our analysis of the LAO ability to produce lateral nanostructures in ferromagnetic semiconductor layers, namely in the thin GaMnAs layers grown by the Molecular Beam Epitaxy (MBE).

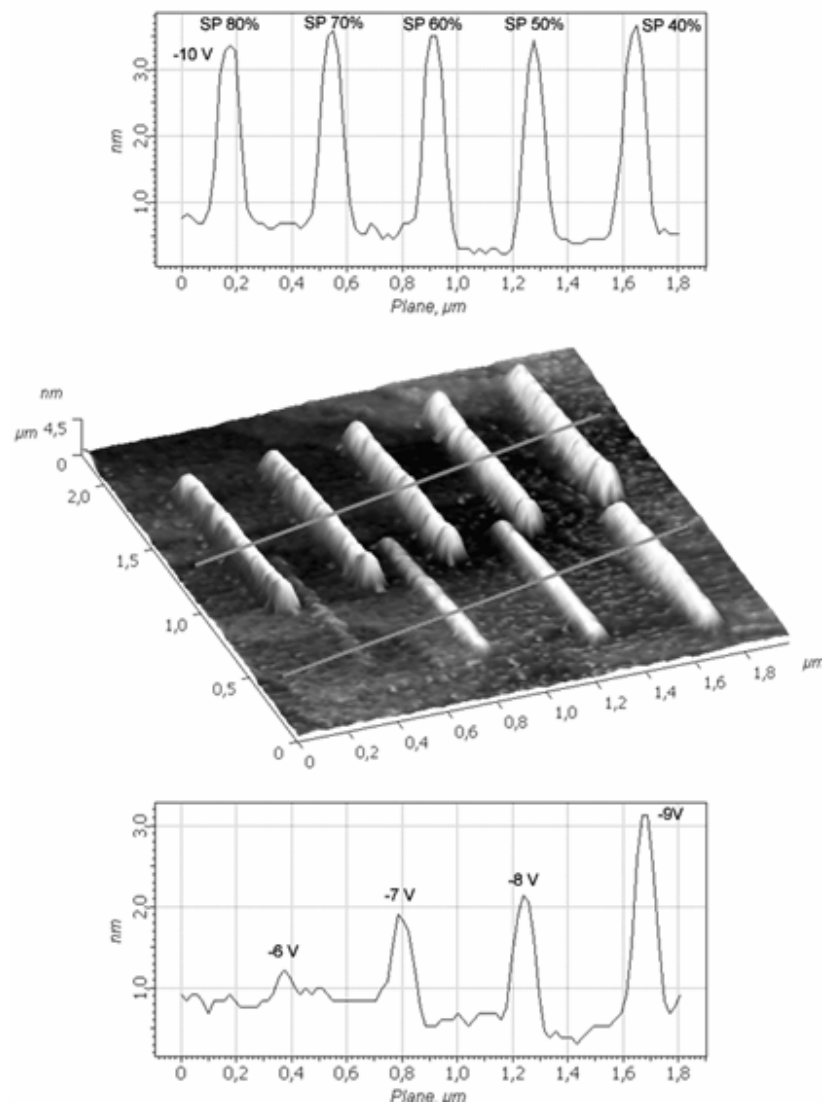

Fig. 2 3D picture of the oxide lines prepared by LAO in the GaMnAs layer using semicontact mode. Set point was lowered to $40 \%$ of original value, increasing the force applied on the tip. Humidity was $75 \%$. Tip velocity during LAO was $400 \mathrm{~nm} / \mathrm{s}$. Negative bias applied on the tip was 5, 6, 7, 8 and $9 \mathrm{~V}$ (from the left) for bottom lines and $10 \mathrm{~V}$ for upper lines, where the tip force was varied without significant effect.

\subsection{MBE Grown GaMnAs Layers}

The layers were prepared by the low temperature (LT) MBE growth in a Veeco Mod Gen II machine using $\mathrm{As}_{4}$ beam. The oxidation was performed on several samples. The first structure consisted of a conventional high temperature GaAs layer of about $200 \mathrm{~nm}$ thick followed by $5 \mathrm{~nm} \mathrm{LT} \mathrm{GaAs} \mathrm{layer} \mathrm{and} 10 \mathrm{~nm}$ GaMnAs layer grown at $200^{\circ} \mathrm{C}$. The surface was atomically smooth with $(1 \times 2)$ reconstruction. The resulting $\mathrm{Ga}_{1-\mathrm{x}} \mathrm{Mn}_{\mathrm{x}} \mathrm{As}$ layer with $\mathrm{x}_{\mathrm{Mn}}$ $=7 \%$ had $\mathrm{T}_{\mathrm{C}}$ of about $55 \mathrm{~K}$ (as grown) and about $150 \mathrm{~K}$ after annealing in air at $200^{\circ} \mathrm{C}$ for 2 hours. After annealing the layer was conducting with the conductivity $200 \Omega^{-1} \mathrm{~cm}^{-1}$. The second sample consists of $200 \mathrm{~nm}$ GaAs buffer layer followed by $4.5 \mathrm{~nm}$ of LT GaAs layer and $50 \mathrm{~nm}$ of GaMnAs. 

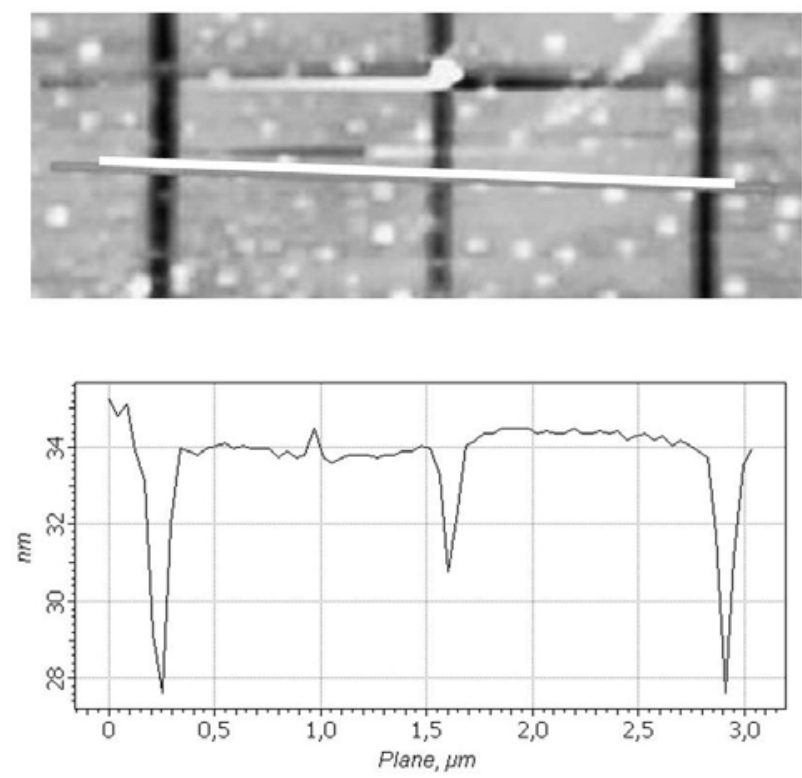

Fig. 3. The AFM picture of three oxide lines after etching in the $\mathrm{HCl}$ (upper) and the 1D profile along the white line (bottom).

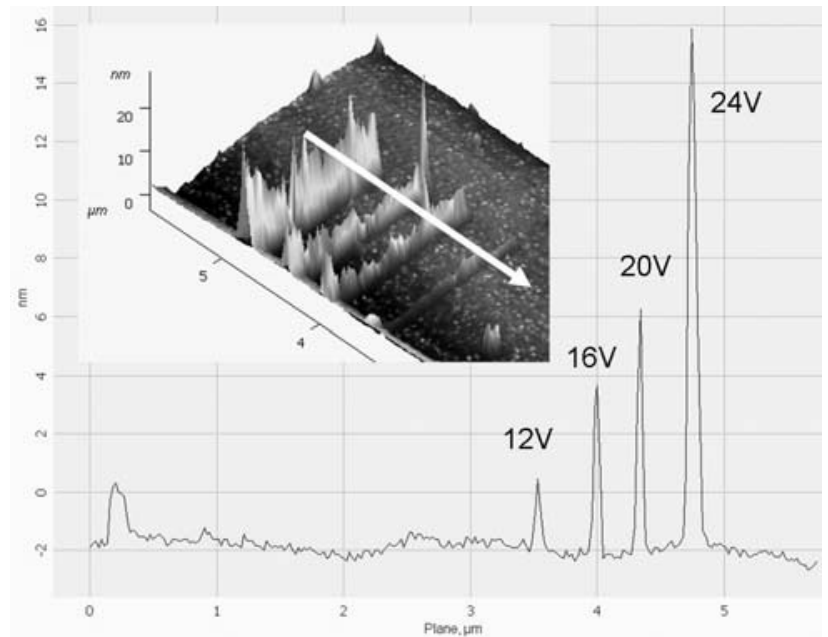

Fig. 4. 1D profile of four oxide lines prepared by LAO in GaMnAs layer with the external voltage source. Set point was lowered to $40 \%$ of original value. Humidity was $75 \%$. Tip velocity during LAO was $400 \mathrm{~nm} / \mathrm{s}$. Negative bias applied on the tip was $12,16,20$ and $24 \mathrm{~V}$. 3D picture with white $1 \mathrm{D}$ section line is in the insert.

\subsection{LAO Parameters and Results}

Several parameters are required to be set correctly for the preparation of homogenous and sufficiently high oxide lines. Ambient humidity is very important for optimal oxidation process [9]. The water condensates in the strong electrical field $\left(10^{10} \mathrm{Vm}^{-1}\right)$ between the tip and the sample. Due to capillary forces condensated water creates

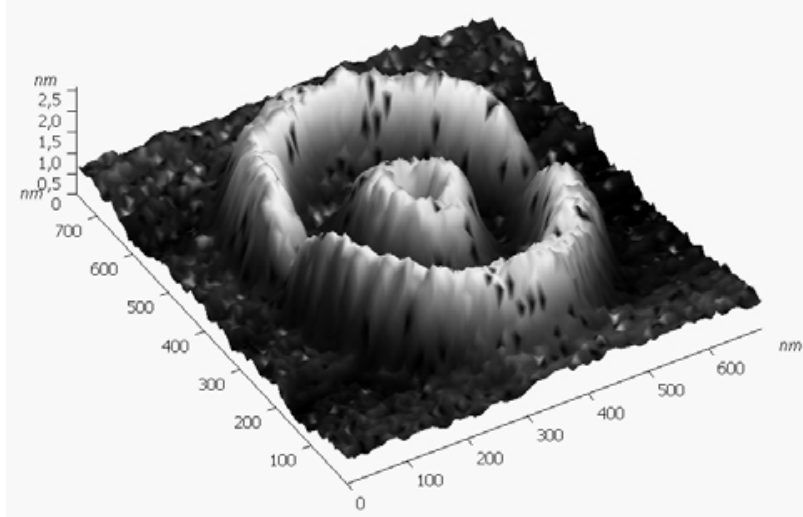

Fig. 5. The Corbino ring with the diameter $200 \mathrm{~nm}$ produced by two LAO circles.

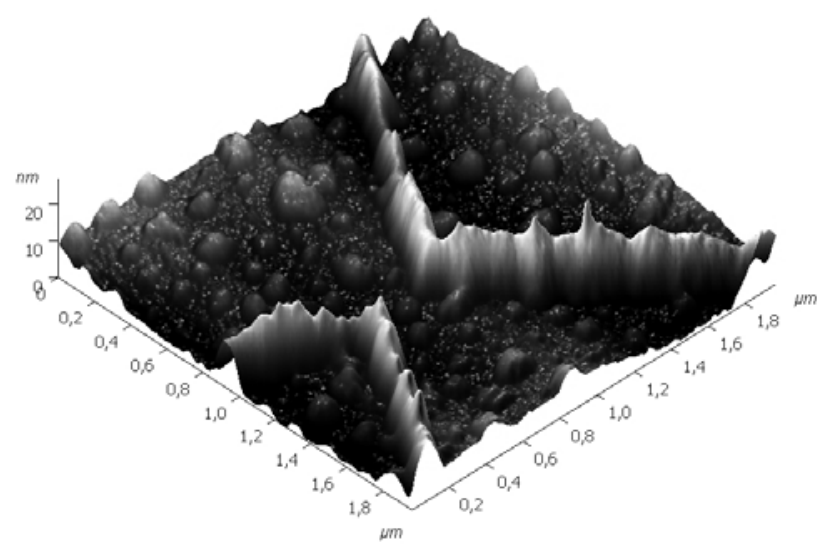

Fig. 6. The 1D constriction realized by two LAO lines in the centre of Hall bar.

a water junction, which sequentially dissociates and acts as electrolyte for following oxidation. The LAO process was performed with the AFM microscope Smena NTMDT placed in the sealed box with the controlled humidity in the range $45-80 \%$. The oxide was grown in the semi-contact mode of the AFM. Sample was positively biased with respect to the AFM tip with the bias from 6 to $24 \mathrm{~V}$. The conductive diamondcoated AFM tips with the radius $30 \mathrm{~nm}$ were used for the oxidation. The tip speed was changed from $200 \mathrm{~nm} / \mathrm{s}$ to $2 \mu \mathrm{m} / \mathrm{s}$ during the oxidation. We used external voltage source to increase the applied bias above 10V. In the Fig. 2 the oxide lines prepared by LAO using internal voltage source are shown. Set point was lowered to $40 \%$ of original value, increasing the force applied on the tip. Humidity was kept at $75 \%$. Tip velocity during LAO was $400 \mathrm{~nm} / \mathrm{s}$. Negative bias applied on the tip was 5, 6, 7, 8 and $9 \mathrm{~V}$ for bottom lines and $10 \mathrm{~V}$ for upper lines, where the tip force was varied without significant effect. 


\section{J. Voves, Z. Šobán̆, M. Jelínek, V. Komarnicki, M. Cukr, V. Novák \\ Nanostructures defined by the local oxidation of ferromagnetic GaMnAs layer}

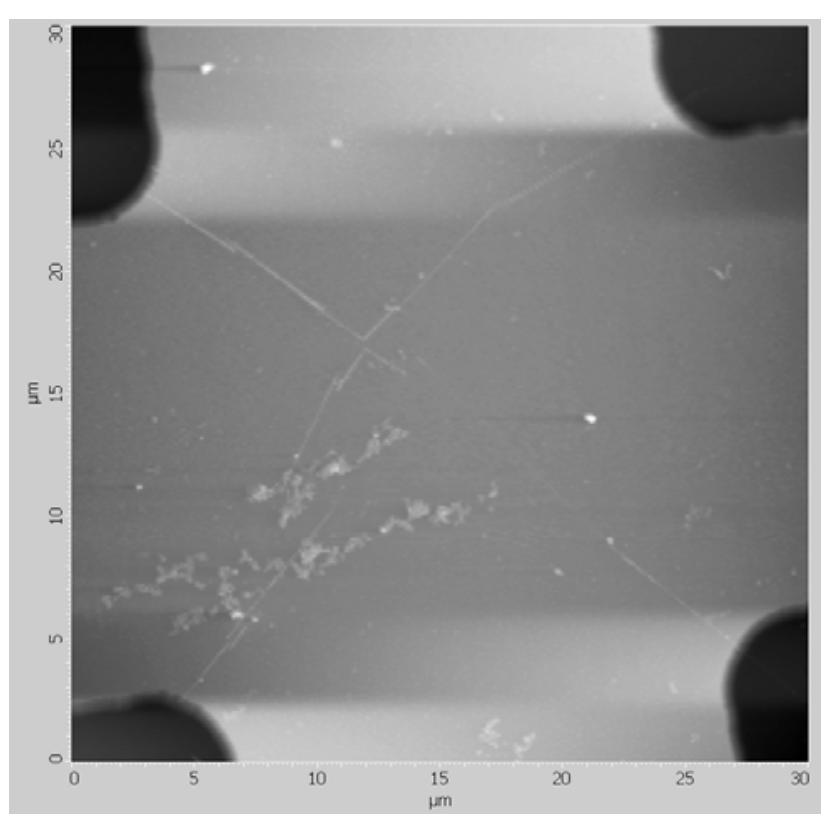

Fig. 7. AFM picture of the Hall bar centre with the $20 \mu \mathrm{m}$ width.

One sample was etched in the $3 \% \mathrm{HCl}$ after the oxidation. Resulting profile shows, that the oxide depth in the susbstrate is approximately the same as the line height above the surface (Fig. 3). In the Fig. 4 four oxide lines are shown prepared by LAO with the external voltage source. Set point was lowered to $20 \%$ of original value on the second sample. Humidity was $50 \%$. Tip velocity during LAO was $500 \mathrm{~nm} / \mathrm{s}$. Negative bias applied on the tip was 12,16, 20 and $24 \mathrm{~V}$. The maximum height of the line is reached for the bias $24 \mathrm{~V}(18 \mathrm{~nm})$. Different optimal oxidation speed of $400 \mathrm{~nm} / \mathrm{s}$ and ambient humidity of $75 \%$ have been found for the each sample. No significant change in the line height and homogenity has been observed by the tip speed up to $1 \mu \mathrm{m} / \mathrm{s}$.

\section{SIMULATION}

The micromagnetic modeling can predict the magnetic behavior of materials on a short length scales of the order of nanometers. Individual magnetic moments will want to align with an external field, until the local energy minimum is reached. If the external field changes, the moments will rotate and the domain patterns change and move to a new configuration, corresponding to the new local energy minimum. The main focus of micromagnetism is to study the magnetization reversal process and the complex domain configurations, especially in thin films.

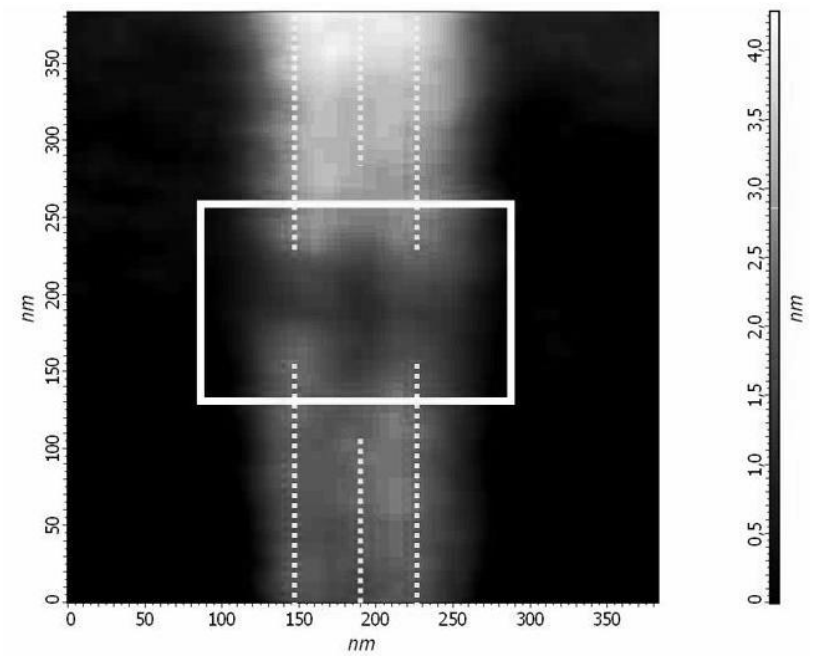

Fig. 8. The quantum dot produced by three interrupted lines with $38 \mathrm{~nm}$ spacing (dotted white lines). Simulated structure is defined by the white rectangle.

\subsection{Micromagnetic Simulator}

The micromagnetic Simulator SimulMag [10] is an easyto-use PC-based magnetic design tool. The simulator allows one to simulate the system response of a magnetic device or circuit under the influence of external magnetic fields, currents, or local field sources. The analysis is based on a collection of single-domain elements whose size and position can be specified by the designer. The elements may be magnetic or nonmagnetic, a conductor, or an insulator. The element's magnetic properties, such as magnetization, anisotropy, pinning fields, and resistivity must be specified or selected from a library of materials. Electrical circuits can be specified and currents applied. The elements interact through magnetostatic interactions and user specified exchange interactions. The elements are arranged in groups to allow different structures to be built up and manipulated independently. Relative motion between the groups can be specified.

\subsection{Simulated Structure}

Three-dimensional nanostructure with two constrictions was approximated by the rectangle shaped oxide regions (rectangle in Fig. 8) constricting thin GaMnAs layer on the semi-insulating GaAs buffer. Analysis of the structure in the external magnetic field with different orientation was performed by means of micromagnetic simulation software SimulMag. This simulator minimizes the Gibbs free energy of the micromagnetic system and uses semi-empirical models for GMR and AMR calculations. Input structure for the 


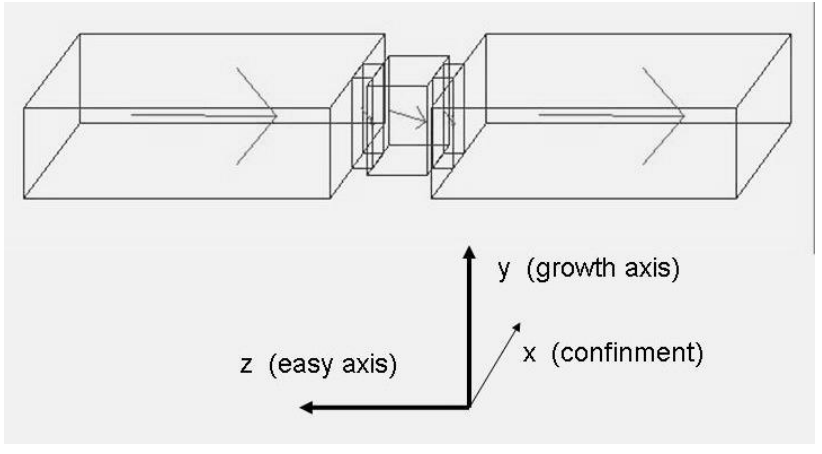

Fig. 9 The input structure for the micromagnetic simulation.

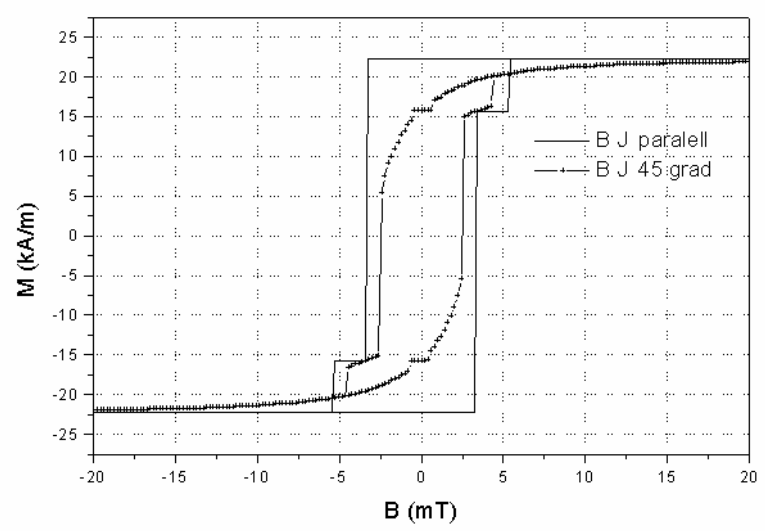

Fig. 10 Results of micromagnetic simulation by the external magnetic field. The field was changed from $30 \mathrm{mT}$ to $-30 \mathrm{mT}$ and back. Resulting magnetisation of the sample shows different levels of hysteresis with different in-plane field orientations (magnetic field B paralell to the current $\mathrm{J}$ and with the angle $45^{\circ}$ between $\mathrm{B}$ and $\mathrm{J}$ ).

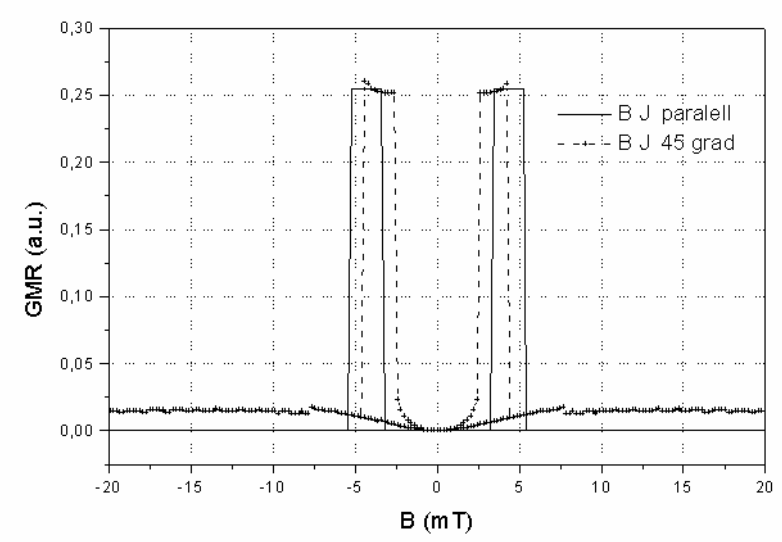

Fig. 11 Qualitative semi-empirical values of GMR with the same magnetic field orientations as in the Fig. 9.

simulation is shown in the Fig. 9. Magnetic easy axis was oriented along the $\mathrm{z}$ direction. The field was changed

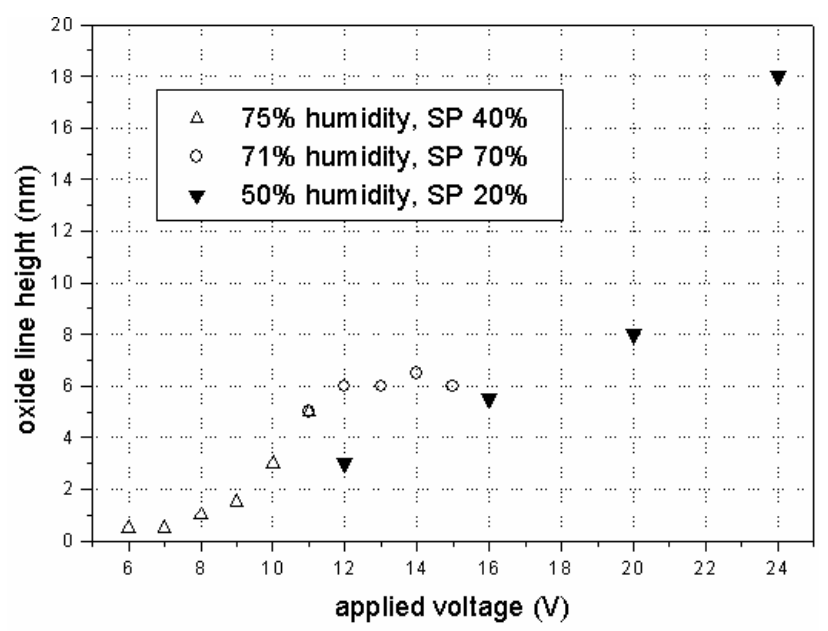

Fig. 12 Oxide line height vs. voltage applied on the AFM tip.

from $30 \mathrm{mT}$ to $-30 \mathrm{mT}$ and back. We performed the static calculation of the structure magnetisation. Saturated magnetisation of the central quantum dot was chosen a little higher $(40 \mathrm{kA} / \mathrm{m})$ than in other regions $(20 \mathrm{kA} / \mathrm{m})$ to simulate the retarded switching of the central part magnetisation during the magnetic field sweep. Resulting magnetisation of the sample shows different levels of hysteresis with different field orientations (Fig. 10) as expected. The hysteresis loops show several nonlinearities due to retarded domain switching of the central dot by the magnetic field change. These nonlinearities produce the sharp GMR changes, as shown in the Fig. 11.

\section{CONCLUSION}

The LAO can be applied to very thin ferromagnetic semiconductor layers to define quantum point contacts, quantum wires, rings, dots and other devices. We analyzed the influence of the different conditions of LAO by AFM tip applied on the thin ferromagnetic GaMnAs layer. The maximum height of the obtained oxide lines was $18 \mathrm{~nm}$ by $24 \mathrm{~V}$ applied on the layer in respect to the AFM tip (Fig. 12). Oxidation speed of $400-500 \mathrm{~nm} / \mathrm{s}$ and ambient humidity of $50-75 \%$ have been used. Optimal parameters should be found separately for the each GaMnAs layer in the dependence of its resistivity and the surface quality. Nanostructures with the quantum dot in between two constrictions were prepared. The analysis of the prepared nanostructure by means of micromagnetic simulation software SimulMag was performed. The simulation can be used for the qualitative estimation of the ferromagnetic nanostructure behavior. Low temperature magnetotransport experiments are started for the further analysis of LAO patterned ferromagnetic nanostructures. 


\section{ACKNOWLEDGEMENT}

The work was supported by the grant No. 102/06/0381 GACR, by the grant No. KAN400100652 GA CAS and by the grant No. MSM 6840770014 The Ministry of Education CR.

\section{REFERENCES}

[1] J. A. Dagata, J. Schneir, H. H. Harary, C. J. Evans, M. T. Postek, J. Bennett, "Modification of hydrogen-passivated silicon by a scanning tunneling microscope in air", Appl. Phys. Lett. 56, p. 2001, 1990

[2] B. Irmer, M. Kehrle, H. Lorenz, and J. P. Kotthaus, „Fabrication of $\mathrm{Ti} / \mathrm{TiO} \mathrm{x}$ tunneling barriers by tapping mode atomic force microscopy induced local oxidation", Appl. Phys. Lett., vol. 71, pp. 1733-1735, 1997

[3] J. Červenka, R. Kalousek, M. Bartošík, D. Škoda, O. Tomanec, T. Šikola, "Fabrication of nanostructures on $\mathrm{Si}(100)$ and GaAs(100) by local anodic oxidation", Applied Surface Science, vol. 253, pp. 2373-2378, 2006

[4] R. Nemutudi, N.J. Curson, N.J. Appleyard, D.A. Ritchie, G.A.C. Jones, "Modification of a shallow 2DEG by AFM lithography", Microelectronic Engineering, vol. 57-58, pp. 967-973, 2001
[5] U. F. Keyser, H. W. Schumacher, U. Zeitler, and R. J. Haug, K. Eberl, "Fabrication of a single-electron transistor by current-controlled local oxidation of a two-dimensional electron system", Appl. Phys. Lett., vol. 76, pp. 457-459, 2000

[6] A. Pakes, P. Skeldon, G. E. Thompson, J. W. Fraser, S. Moisa, G. I. Sproule, M. J. Graham, S. B. Newcomb, "Anodic oxidation of gallium nitride", Journal of Materials Science, vol. 38, pp. 343-349, 2003

[7] C. Rüster, T. Borzenko, C. Gould, G. Schmidt, and L.W. Molenkamp, X. Liu, T. J.Wojtowicz, J. K. Furdyna, Z.G. Yu, M. E. Flatte,"Very Large Magnetoresistance in Lateral Ferromagnetic (Ga,Mn)As Wires with Nanoconstrictions", 91, p. $216602-1,2003$

[8] A. D. Giddings, M. N. Khalid, T. Jungwirth, J.Wunderlich, S. Yasin, R. P. Campion, K.W. Edmonds, J. Sinova,

K. Ito, K.-Y. Wang, D. Williams, B. L. Gallagher, C. T. Foxon, "Large Tunneling Anisotropic Magnetoresistance in (Ga,Mn)As Nanoconstrictions", Phys. Rev. Lett., vol. 94, p. 127202, 2005

[9] M. Bartošík, D. Škoda, O. Tomanec, R. Kalousek, P. Jánský, J. Zlámal, J. Spousta, T. Šikola, "The influence of humidity on the kinetics of local anodic oxidation", Journal of Physics: Conference Series., vol. 61, pp. 75-79, 2007

[10] http://math.nist.gov/oomf/contrib/simulmag 